CAHIERS DE

NARRATOLOGIE

\section{Cahiers de Narratologie}

Analyse et théorie narratives

$30 \mid 2016$

Street Art 2

\title{
Quand les murs de béton muets se transforment en un carnaval de couleur
}

Le street art comme stratégie de résistance sociale contre le modèle commercial de la visibilité

\section{Andrea Baldini}

\section{(2) OpenEdition}

\section{Journals}

Electronic version

URL: http://journals.openedition.org/narratologie/7469

DOI: 10.4000/narratologie.7469

ISSN: 1765-307X

Publisher

LIRCES

\section{Electronic reference}

Andrea Baldini, "Quand les murs de béton muets se transforment en un carnaval de couleur », Cahiers de Narratologie [Online], 30 | 2016, Online since 28 July 2016, connection on 19 April 2019. URL : http:// journals.openedition.org/narratologie/7469; DOI : 10.4000/narratologie.7469

This text was automatically generated on 19 April 2019

Article L.111-1 du Code de la propriété intellectuelle. 


\section{Quand les murs de béton muets se transforment en un carnaval de couleur}

Le street art comme stratégie de résistance sociale contre le modèle commercial de la visibilité

\section{Andrea Baldini}

\section{Introduction}

1 Au cours des 20 dernières années, le nombre de bourses octroyées dans le domaine du street art a observé une forte croissance. Toutefois, malgré l'intérêt interdisciplinaire pour le sujet, il semble toujours impossible d'arriver à un consensus en ce qui concerne sa définition. Le street art affecte le quotidien de nombre de personnes, car il modifie le paysage urbain et tend à susciter la controverse. Par conséquent, tenter de formuler une définition (pratique) du street art n'est pas un simple exercice académique : il s'agit d'une discussion relevant de l'ordre public. Une telle définition peut, certes, se révéler utile dans les débats au sujet des polémiques suscitées par le street art ainsi que pour informer les politiques relatives au développement urbain. Dans cet article, nous avons pour objectif de fournir une définition du street art susceptible de satisfaire aux critères susmentionnés.

2 Nous suggérons que les tentatives précédentes d'élaboration d'une définition, telle celle de Riggle (2010), ont de façon générale ignoré la valeur intrinsèquement subversive de cette forme d'art, bien qu'elles se soient efforcées, à juste titre, de souligner le lien entre le street art et la ville. En négligeant cet aspect, ces tentatives n'ont pas su capturer intégralement la capacité du street art à transformer l'utilisation actuelle de l'espace urbain public, en tirant parti sa présence contestée et en encourageant les passants à imaginer d'autres modes de vie, plus inclusifs, au sein des villes modernes.

3 Par conséquent, nous proposons dans cet article une définition du street art basée sur sa subversivité essentielle, qui tient compte des conséquences de ses fonctions politiques et 
sociales. Nous nous basons sur la pensée d'Irvine (2012) pour argumenter que les œuvres de street art ont entièrement détourné les normes usuelles de visibilité dans les espaces publics. Les artistes de street art défient ces normes en exhibant des stratégies carnavalesques, telles que l'ironie et le détournement (Scott 1990). En ce sens, le street art défie intrinsèquement les hiérarchies dominantes de la visibilité, par le biais de stratégies carnavalesques. À ce titre, les exemples de street art constituent des actes de résistance sociale et politique allant à l'encontre de l'usage des espaces urbains visibles par les hiérarchies dominantes - notamment les hiérarchies de nature commerciale. En s'opposant à la présence dominatrice de la publicité sur les murs des espaces urbains, les œuvres de street art revendiquent, une rue à la fois, la possibilité de s'exprimer en public pour ceux qui n'en ont généralement pas le droit.

La définition que nous proposons insiste sur la subversivité urbaine du street art, tout en soulignant au moins deux dimensions pragmatiques. Premièrement, cette définition rejette la séparation moderne entre l'art et la vie, car elle considère le street art comme un acteur de la lutte politique pour la visibilité. Deuxièmement, étant donné que cette définition reconnaît le rôle du street art dans notre vie quotidienne, elle présente l'appréciation de cet art comme un phénomène qui dépasse les limites étroites de la contemplation désintéressée associée à certaines formes de plaisir.

5 La section 2 analyse certaines considérations qui permettent de justifier, sur le plan métathéorique, le type de définition auquel nous accordons préséance dans le présent article: la définition évaluative. La section 3 revendique que les œuvres défiant les hiérarchies dominantes de la visibilité dans les espaces publics appartiennent au street art. La section 4 analyse et explique la dimension carnavalesque distinctive de cet art « résistant », afin de mettre en lumière son rôle incontestable sur la scène de la résistance sociale. Cette section nous permet, notamment, d'établir notre propre théorie sur la différence entre le street art et l'art public (officiel).

\section{Quelques considérations métathéoriques}

6 Tout d'abord, il est nécessaire de répondre à la question suivante, afin d'élaborer une définition pratique du street art : à quelles questions une définition du street art doit-elle répondre? Appelons cela la question métathéorique. La nécessité de répondre à la question métathéorique fait suite à une simple reconnaissance du pragmatisme intrinsèque et de la nature provisionnelle des tâches définitionnelles. Dans le cadre de l'étude d'un phénomène humain spécifique ou d'une pratique culturelle particulière - notamment, un phénomène aussi ambigu que celui du street art - il est très probable que plusieurs méthodes permettent d'en définir les limites. En outre, il ne sera probablement pas possible d'identifier une seule et unique méthode valide : il se peut qu'il soit nécessaire d'établir différentes définitions qui satisferont à différents objectifs. Par conséquent, il est indispensable de répondre à la question métathéorique, afin de trouver la voie (provisoire) à suivre.

7 La philosophie anglo-américaine de l'art nous procure un appareil conceptuel utile, susceptible de nous aider à répondre à la question métathéorique. En général, les définitions de l'art se divisent en deux catégories: les définitions classificatoires et évaluatives. Les définitions classificatoires de l'art sont descriptives et visent à établir une congruité référentielle. En d'autres termes, leur objectif est d'englober l'intégralité ou la majorité des choses que nous considérons intuitivement comme de «l'art», tout en 
excluant l'intégralité ou la majorité des choses que nous ne considérons pas intuitivement comme de " l'art ${ }^{1}$ ». Par conséquent, une définition classificatoire du street art inclut l'intégralité ou la majorité des choses que nous considérons intuitivement comme du « street art».

$8 \mathrm{Au}$ contraire, les définitions évaluatives de l'art cherchent à fournir une explication de type évaluatif, c'est-à-dire une explication capable de souligner les raisons pour lesquelles les individus aiment créer et apprécier les œuvres d'art ${ }^{2}$. Par conséquent, ce type de définition se soucie moins de la reconnaissance intuitive de ce que l'on considère comme de «l'art»; il est plus normatif que descriptif. Dans ce cas, l'objectif primaire d'une définition évaluative du street art est de déterminer les raisons pour lesquelles les artistes de street art accordent de la valeur à la création de leurs œuvres et d'identifier les raisons qui poussent les amateurs de street art à apprécier ces œuvres.

Dans la section suivante, nous nous attachons à développer une définition évaluative du street art. Commençons par citer trois raisons pour lesquelles nous préférons le type évaluatif au type classificatoire. Premièrement [selon l'argumentation de Matravers (2000)], la nature purement descriptive des définitions classificatoires n'offre aucun indice permettant de justifier l'existence de l'art. Pourtant, cette pratique - dont l'objectif est de présenter des objets à un public - semble nécessiter une telle justification. Dans le cas du street art, dont l'intrusion dans l'espace public est fréquemment controversée, une telle justification paraît impérative. Elle est d'autant plus importante qu'elle doit faire face à ceux qui s'y opposent. Nous sommes d'avis qu'une définition utile du street art, capable d'informer le débat de société, doit pouvoir justifier l'existence de cette pratique artistique.

10 Deuxièmement, et conformément à l'argumentation de Zangwill (2007) et Margolis (2010), bien que les définitions classificatoires semblent également se baser sur des critères évaluatifs, l'inverse n'a pas été observé. Ainsi, au lieu de paraître descriptives, les idées sur lesquelles se basent les définitions classificatoires semblent résulter d'hypothèses évaluatives non vérifiées, visant à distinguer l'art de ce qui n'en est pas. Par conséquent, les questions évaluatives paraissent plus fondamentales que les questions classificatoires. Nous semblons préférer ancrer nos théories artistiques au lieu d'accommoder les cas ambigus, dont nous nous soucions uniquement lorsque la supériorité de la définition évaluative a été établie.

11 En fin de compte, l'un des éléments les plus importants soulignés par Shusterman (2000) concerne l'état pragmatique inerte des définitions: c'est-à-dire qu'elles acceptent la pratique dans son état actuel. Elles semblent inutiles dans le cadre de l'élaboration de considérations heuristiques visant à remettre la pratique sur la bonne voie, en cas de faux pas ou de conflits en tout genre - notamment avec d'autres pratiques ou avec une partie importante de la population. Dans le cas du street art, les deux situations semblent s'appliquer. Premièrement, nombreux sont ceux qui considèrent le street art comme un art dégénératif (Abbey-Lambertz 2012). Deuxièmement, le conflit qui oppose le street art au système juridique et la réception souvent hostile qu'il reçoit de la part de population au sens large sont des caractéristiques inhérentes à cette pratique. Étant donné que l'objectif de cette étude est de fournir une définition composée de considérations heuristiques susceptibles d'avoir un impact positif sur la résolution de ces problèmes, nous donnerons préséance à une définition évaluative. Dans la section suivante, nous soutenons que la subversivité d'une œuvre permet de déterminer son appartenance au street art. 


\section{La subversivité du street art}

12 Il est évident que la majeure partie des œuvres de street art tend à exhiber une subversivité explicite, en ce qu'elle transmet un message clair de contestation politique. Porteuses d'un message politique et social, les œuvres de street art jouent généralement un rôle primordial dans les mouvements de résistance aux quatre coins du monde. Certaines de ces œuvres sont des exemples emblématiques de cette forme d'expression artistique et occupent une place importante dans l'histoire de l'art. Les œuvres de nombreux artistes du street art, tels que Banksy, Blu, ROA, et Ron English, sont généralement à caractère politique. On ne compte plus le nombre d'œuvres politiquement chargées et les mouvements de lutte pour le changement politique et social en Europe, en Afrique et en Asie qui exploitent fréquemment le street art comme outil de contestation (Baldini 2015; Bartolomeus 2012; Hamdy and Stone 2014; ryanbyrne123 2015 ; Siregar 2012).

13 Toutefois, la plupart des exemples populaires de street art ne contiennent pas de messages politiques explicitement subversifs. Considérons, par exemple, le Manhattan Tag de MOMO ou les paysages géométriques d'Ethn!k.

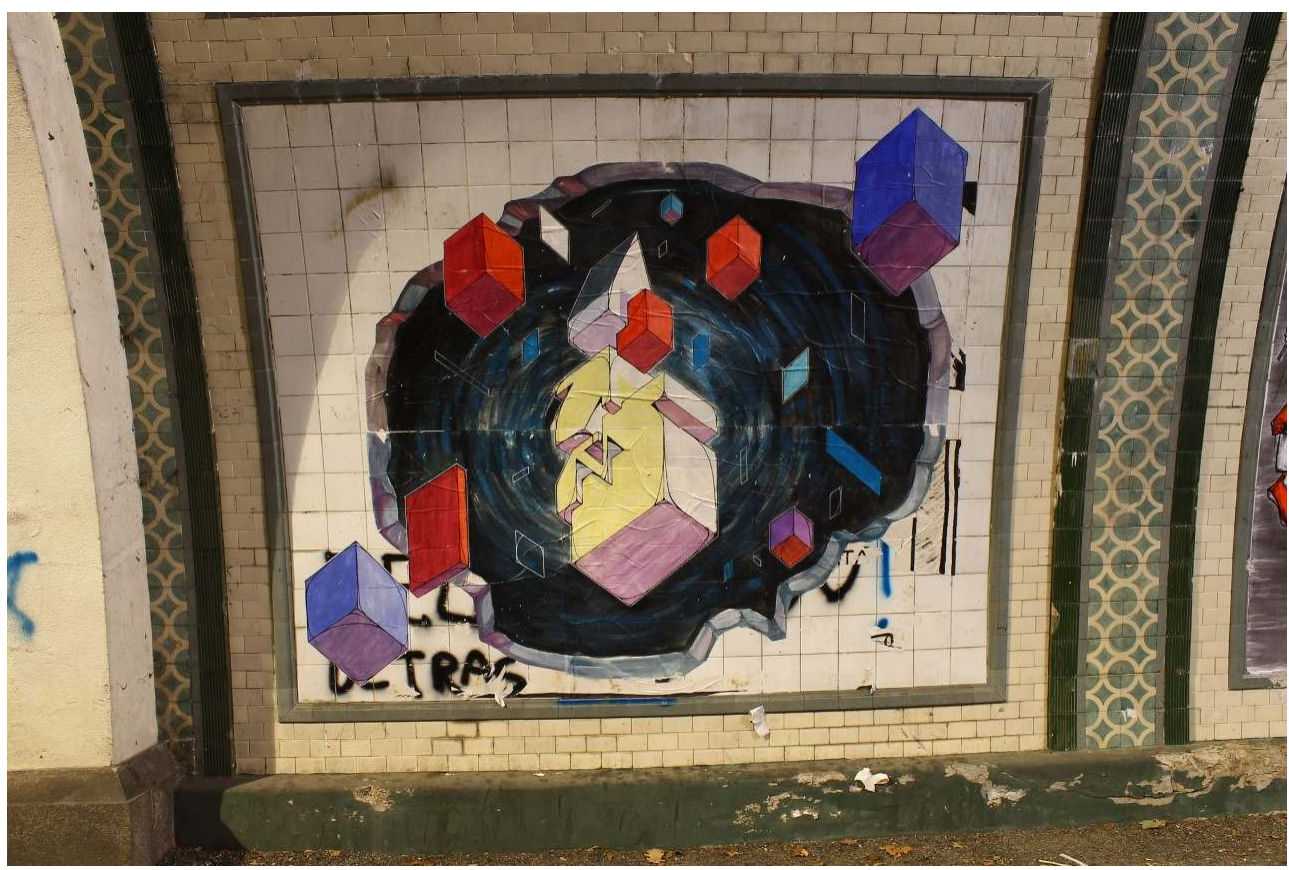

«Une œuvre d'Etn!k réalisée durant le Shit Art Fair. (etnik par Omino 71, https://www.flickr.com/ photos/omino71/)»

14 Quel était donc le message politique dans la fine ligne de peinture jaune que MOMO utilisait pour écrire son nom à une échelle monumentale, sur les trottoirs des rues de New York? De même, le paysage géométrique flottant, onirique, que l'on retrouve dans plusieurs des peintures murales d'Etni!k, ne constitue pas de message clair de protestation. Cependant, les œuvres de street art (y compris celles qui ne sont pas politiquement chargées, telles celles de MOMO et d'Etn!k) sont subversives dans un sens plus profond.

15 Afin de pouvoir expliquer la profondeur et le pouvoir subversif fondamental du street art, commençons par introduire la notion de " partage du sensible ${ }^{3} »$. Nous empruntons cette 
notion à l'excellente explication de Jacques Rancière, concernant la relation entre l'espace public et la politique ${ }^{4}$. Avec le partage du sensible, Rancière indique entre autres, un ensemble de normes et de lois sociales permettant de réglementer le visible dans les espaces publics d'une société donnée. Les sociétés actuelles se basent sur des conventions sociales et des ordonnances légales pour distinguer ces formes étranges d'expression de contenus qui peuvent ou ne peuvent pas être exposés en public. À titre d'exemple, la visibilité de contenu pornographique est censurée dans la plupart des sociétés contemporaines, ce qui a généralement pour objectif d'interdire l'exposition publique d'images à caractère sexuel.

En faisant référence à Baker (2007), de Certeau et Giard (1998), et Mattelart (1991), nous souhaitons suggérer que les lois et les normes sociales qui réglementent la visibilité dans les villes modernes constituent un type spécifique de partage du sensible. Un tel partage offre une place de premier plan à la communication commerciale, permettant aux campagnes publicitaires de dominer le paysage urbain (Iveson 2012). Autant désigner ce phénomène comme un partage du sensible basé sur le régime de visibilité d'entreprise - ou, tout simplement le « régime d'entreprise ».

17 Le régime d'entreprise a profondément transformé la nature et l'utilisation de l'espace public. Rassemblant autrefois des lieux et sites propices aux rencontres informelles, les places et les rues d'aujourd'hui sont généralement réduites à des emplacements publicitaires. En attirant l'attention des passants avec leurs affiches, ces places offrent moins de possibilités d'interaction sociale authentique: il ne reste aux communautés urbaines qu'un vestige de socialités.

18 Il est essentiel de reconnaître à quel point le régime d'entreprise façonne la nature et l'utilisation des espaces publics, pour comprendre la dimension subversive du street art. Il suffit de se référer à l'argumentation convaincante d'Irvine (2012) pour comprendre qu'en introduisant des contenus et des formes visuels inattendus dans le paysage urbain, le street art produit "une contre-imagerie ${ }^{6}$ » qui permet d'interroger la présence dominante de la publicité dans les espaces publics. En ce sens, le pouvoir subversif du street art est lié à sa capacité à s'approprier les rues et les places publiques, de sorte à déstabiliser le régime d'entreprise.

19 L'hypothèse d'Irvine est prometteuse et affecte significativement la définition du street art. Développons cet argument davantage. En tant que formes expressives dont le but est de défier le régime d'entreprise, les œuvres de street art dérangent ponctuellement et profondément la nature et l'utilisation des espaces urbains, qui cessent temporairement de servir de simples surfaces publicitaires pour se transformer en vitrines d'œuvres libres. Une telle transformation perturbe également les attentes des passants, qui répondent à ces « invasions de l'espace urbain » par différentes réactions - du choc à la gaieté, en passant par la colère ou le dégoût. Les spectateurs partagent généralement leurs réactions aux œuvres de street art avec les autres ; par conséquent, dans ce contexte précis, ces œuvres sont des catalyseurs de dialogue et d'échange.

20 Outre la description "phénoménologique » suggérée ci-dessus, nous proposons trois arguments complémentaires en vue de soutenir l'hypothèse selon laquelle le street art est essentiellement subversif'. Tout d'abord, l'hypothèse suggérant que l'objectif principal du street art est de remettre en question ce que nous appelons le régime d'entreprise est répandue parmi les artistes de street art. Young (2014) signale que la majorité des artistes de street art avec lesquels elle s'est entretenue cherchent à mettre en cause la présence dominante de la publicité dans les espaces publics. Entre autres, Ron English, Shepard 
Fairey, et Banksy reconnaissent clairement que leurs œuvres leur permettent de s'opposer à la transformation des espaces publics en emplacements publicitaires ${ }^{8}$. Par exemple, lorsque Banksy s'interroge sur la question du street art en tant que forme de vandalisme, il explique que les «personnes qui défigurent vraiment le paysage qui nous entoure sont les sociétés qui étalent leurs slogans géants sur tous les immeubles.... [E]lles ont commencé à livrer cette bataille et nous avons choisi d'utiliser les surfaces murales pour nous défendre ${ }^{9}$ ». À cet égard, une analyse des intentions des artistes de street art confirme le caractère spécifique de sa subversivité, comme indiqué dans le présent article.

21 Deuxièmement, les œuvres basées sur un style urbain (graffiti, pochoir urbain, etc.), visant à faire la promotion de produits commerciaux sont généralement considérées - si elles font l'objet d'une considération quelconque - comme de fausses œuvres de street art. Confino définit ce type de publicité comme de «fausses œuvres de street $\operatorname{art}^{10}$ ». D'ailleurs, il emprunte les mots du militant environnementaliste L. Hunter Lovins pour indiquer très clairement le manque de valeur de cette forme de communication : " le faux street art, c'est complètement nul ${ }^{11}$ ". A cet égard, le cas des Vespa Squareheads de Fauxreel est instructif. Fauxreel, dont le vrai nom est Dan Bergeron, est un artiste de street art connu pour son «style graffiti virtuel ». En 2008, il s'est associé à Vespa et a illégalement installé 324 affiches publicitaires de $2 \mathrm{~m}$ de haut dans les rues de Toronto - à savoir, les Vespa Squareheads. Bien que ces œuvres aient des similarités avec les autres œuvres de Fauxreel sur les plans visuel et stylistique, les amoureux du street art ont refusé de donner à ces affiches le statut de street art (Vartanian 2010). En outre, cette trahison purement commerciale a poussé certaines personnes à s'interroger sur les œuvres précédentes de Fauxreel (Simoes 2008 ; Walker 2008). Le résultat est le suivant : lorsqu'un travail ne s'oppose pas au régime d'entreprise, il ne peut pas être considéré comme du street art. En effet, une œuvre de street art n'est pas simplement définie par des caractéristiques esthétiques spécifiques ou un style particulier. Une œuvre de street art joue un certain rôle dans l'espace public - un rôle de nature essentiellement subversive, qui remet en question l'utilisation commerciale des surfaces urbaines visibles.

L'argument final se base sur une idée largement répandue au sujet du street art et de ses liens avec le monde artistique. Nombreux sont ceux qui en conviennent: le street art existe et se développe en opposition avec les institutions artistiques officielles (Bengtsen 2015 ; Riggle 2010). Cependant, s'il est dépourvu de sa subversivité, le street art est facilement réabsorbé par ledit monde artistique officiel. Par conséquent, c'est l'identité même de cette forme d'art qui est remise en question. Un phénomène similaire affecte le street art en Chine continentale. À titre d'exemple, Volodzko (2015) qualifie les œuvres urbaines chinoises généralement associées au street art (p. ex., graffiti, pochoir urbain, etc.) de «bénignes ». Le manque de subversivité dans les œuvres urbaines chinoises résulte de plusieurs facteurs. Énumérons donc les trois facteurs qui seront considérés comme les plus importants dans le cadre de cet article. Pour commencer, les artistes chinois craignent les représailles et évitent donc de créer des œuvres controversées. Ensuite, en raison de la taille impressionnante des métropoles chinoises, une grande partie du paysage urbain chinois auquel les artistes ont accès est dépourvue de communication commerciale (Thibault 2014). Il est donc relativement aisé de créer des œuvres qui n'entrent pas en conflit avec le régime d'entreprise. Enfin, les écritures murales sont répandues en Chine, où elles font historiquement partie de la culture. Le peuple chinois ignore souvent leur présence et ne considère pas les graffitis ou les œuvres 
similaires comme un acte de défiance envers le régime d'entreprise (Pan 2014 ; Valjakka 2013). Compte tenu de leur manque de subversivité, les styles urbains chinois sont considérés comme des tendances appartenant à l'art chinois officiel. Reconnu comme le premier artiste de street art chinois, Zhang Dali a récemment critiqué le statut de l'art urbain chinois. De son point de vue, le street art « est actuellement à la mode en Chine et a perdu sa valeur d'art contestataire ${ }^{12} »$. Une telle assimilation semble compromettre significativement l'appartenance au street art de l'art urbain chinois.

Pour toutes les raisons susmentionnées, l'hypothèse selon laquelle une œuvre peut être considérée comme du street art si elle s'oppose au régime d'entreprise semble justifiée. N'oublions pas de souligner ce qui différencie la définition présentée dans cet article des autres définitions du street art, notamment celle défendue par Riggle ${ }^{13}$ (2010). Riggle (2010) développe de façon intéressante l'idée - raisonnable - selon laquelle le street art est créé « dans les rues » («Urban Dictionary: Street Art » 2015). Il suggère qu'une œuvre devient du street art lorsqu'elle exploite la rue d'un point de vue artistique intéressant. Ainsi, l'environnement urbain est un élément essentiel à la structure d'une œuvre de street art, car il en influence significativement le sens. Par conséquent, retirer une illustration de street art de son environnement original est susceptible de la détruire.

En reconnaissant la signification de la relation intrinsèque entre le street art et l'espace urbain, la définition de Riggle représente un bon début, bien qu'elle soit loin d'être suffisante. Riggle a raison lorsqu'il écrit que l'utilisation artistique de la rue contribue au sens du street art. C'est l'absence de la notion de réciprocité que nous réfutons dans le présent article: le street art affecte également l'importance des lieux qu'il occupe. Le street art ne se contente pas d'utiliser la rue: il la transforme. Grâce à leur pouvoir subversif sur le plan politique, les œuvres de street art transforment les lieux qu'elles occupent en espaces conflictuels, où les pouvoirs régissant la visibilité dans les villes modernes sont contestés.

S'il perd la capacité de transformer un espace public en espace conflictuel, le street art est dépourvu de son sens et de son statut de street art. Par exemple, Riggle (2010) a sans doute raison lorsqu'il affirme que retirer une œuvre de la rue et la placer dans un espace dédié à la vente d'objets d'art en détruirait définitivement le sens et lui retirerait son statut d'œuvre de street $\operatorname{art}^{14}$. Toutefois, il a tort lorsqu'il prétend que ce changement de sens et de statut résulte uniquement du retrait de l'œuvre de son contexte original. Plus précisément, l'œuvre de street art perd sa force subversive lorsqu'elle est réduite au statut de marchandise. À l'instar des icônes politiques transformées en logos de t-shirt (tel Che Guevara!), elle s'intègre au système qu'elle est censée défier.

26 Dans la section suivante, nous argumenterons que le street art cherche à saboter le régime d'entreprise en déployant des stratégies carnavalesques; l'utilisation de ces stratégies aura des conséquences significatives sur ce qui définit la distinction entre le street art et l'art public (officiel) et sur notre compréhension des possibilités de résistance sociale y étant associées.

\section{Le carnaval du street art}

Bien entendu, il est tout à fait possible de s'opposer à l'argument selon lequel d'autres formes d'art du domaine public sont très bien équipées pour défier les hiérarchies dominantes de la visibilité. Prenons l'exemple de la sculpture d'Allison Leppard, créée par 
Marc Quinn. Chacun se souvient de la sculpture de Quinn comme d'un objet monumental, taillé dans du marbre Carrara et représentant Allison Leppard - une femme handicapée nue et enceinte. L'œuvre était installée sur le quatrième socle de Trafalgar Square, à Londres. En raison de son contenu inhabituel et de son placement stratégique, l'œuvre de Quinn défie profondément les normes de visibilité des espaces publics, car ces derniers n'offrent aucune représentation ou célébration de personnes handicapées. Comme l'artiste l'a un jour fait remarquer, « l'absence de représentations positives de personnes handicapées dans l'histoire de l'art public ${ }^{15}$ » a inspiré son œuvre. En ce sens, il semble que d'autres formes d'art du domaine public - y compris l'art public - puissent remettre en question les normes qui réglementent la visibilité dans les espaces publics.

Il est inutile de nier que des pratiques artistiques autres que le street art peuvent défier les normes actuelles de visibilité dans les espaces publics - c'est-à-dire, ce que nous appelons ici le régime d'entreprise. Ce qui distingue le street art est la nature de son opposition. En d'autres termes, l'utilisation de stratégies carnavalesques par les artistes de street art pour lutter contre le régime d'entreprise est inhabituelle. De telles stratégies incluent l'appropriation et le détournement, l'inversion, l'ironie, la satire et la parodie. Quand bien même distinctes, elles ont toutes un point commun : elles sont carnavalesques dans le sens donné à ce terme par Bakhtin (1984). En y déchaînant un carnaval de couleur, le street art semble animer les murs de béton muets de la ville.

D'après Bakhtin, le carnavalesque est caractérisé par le courage de ceux qui s'opposent aux pouvoirs officiels et aux hiérarchies. En surmontant la peur, les pratiques carnavalesques créent le chaos là où l'ordre règne normalement; elles ridiculisent ainsi "les symboles de pouvoir et de violence [dont] le sens est détourné16». C'est exactement ce que fait le street art, lorsqu'il s'approprie les espaces publics à l'aide d'œuvres peintes et d'autres éléments colorés : il sabote le régime d'entreprise et introduit le chaos là où l'autorité et l'ordre règnent normalement, tout en ridiculisant ceux qui sont au pouvoir. À ce titre, l'artiste-fou devient roi de la rue dans le monde détourné du street art et il rend le droit de s'exprimer en public à ceux qui n'en ont généralement pas le droit ${ }^{17}$.

Cet élément carnavalesque semble être absent (ou presque) d'autres pratiques artistiques du domaine public et notamment de l'art public. En effet, bien que les artistes publics, tel Quinn, défient les normes hiérarchiques de visibilité en introduisant des éléments inattendus, ils ne le font pas de manière carnavalesque. Au lieu de chercher à ridiculiser les normes courantes, ils semblent tenter d'en établir de nouvelles. Cet objectif à long terme ne fait pas partie des préoccupations des artistes de street art, dont les œuvres sont de nature éphémère. Ils n'aspirent pas à établir un nouveau système de visibilité, mais plutôt à saboter celui qui existe.

31 Les chercheurs d'ethnographie et de sociologie qui étudient les graffiti-artists (Brighenti 2010 ; Campos 2013 ; Ferrell 1996 ; Halsey et Young 2006) présentent une argumentation bien étayée en faveur du point de vue détaillé dans cet article, selon lequel le street art est une pratique carnavalesque. Bien que leurs résultats ne soient généralement pas conceptualisés par rapport à la notion de carnavalesque présentée par Bakhtin, ils s'accordent bien avec cette idée. Pour preuve, les mots qu'ils utilisent pour décrire les activités des graffiti-artists sont souvent très proches de ceux utilisés pour parler du carnaval et du carnavalesque. Selon Campos, « les jeunes graffiti-artists [recherchent] une sensation de liberté et d'autonomie, [en] échappant au contrôle disciplinaire des normes sociales et des habitudes de ce monde ${ }^{18} »$. Lors d'un entretien avec Brighenti, le graffitiartist italien Paolins définit son activité comme une "pratique ingouvernable ... mise en 
place par des personnes dont les actes ignorent toutes les règles ${ }^{19}$ ». Dans le même esprit, Fra32, graffiti-artist de renommée internationale, a déclaré: « $\mathrm{Si}$ tu ne peux pas surmonter ta peur de te faire attraper ou de te blesser, tu n'es pas un graffiti-artist.... Si ça ne contrarie pas certains conformistes, ce n'est un graffiti ».

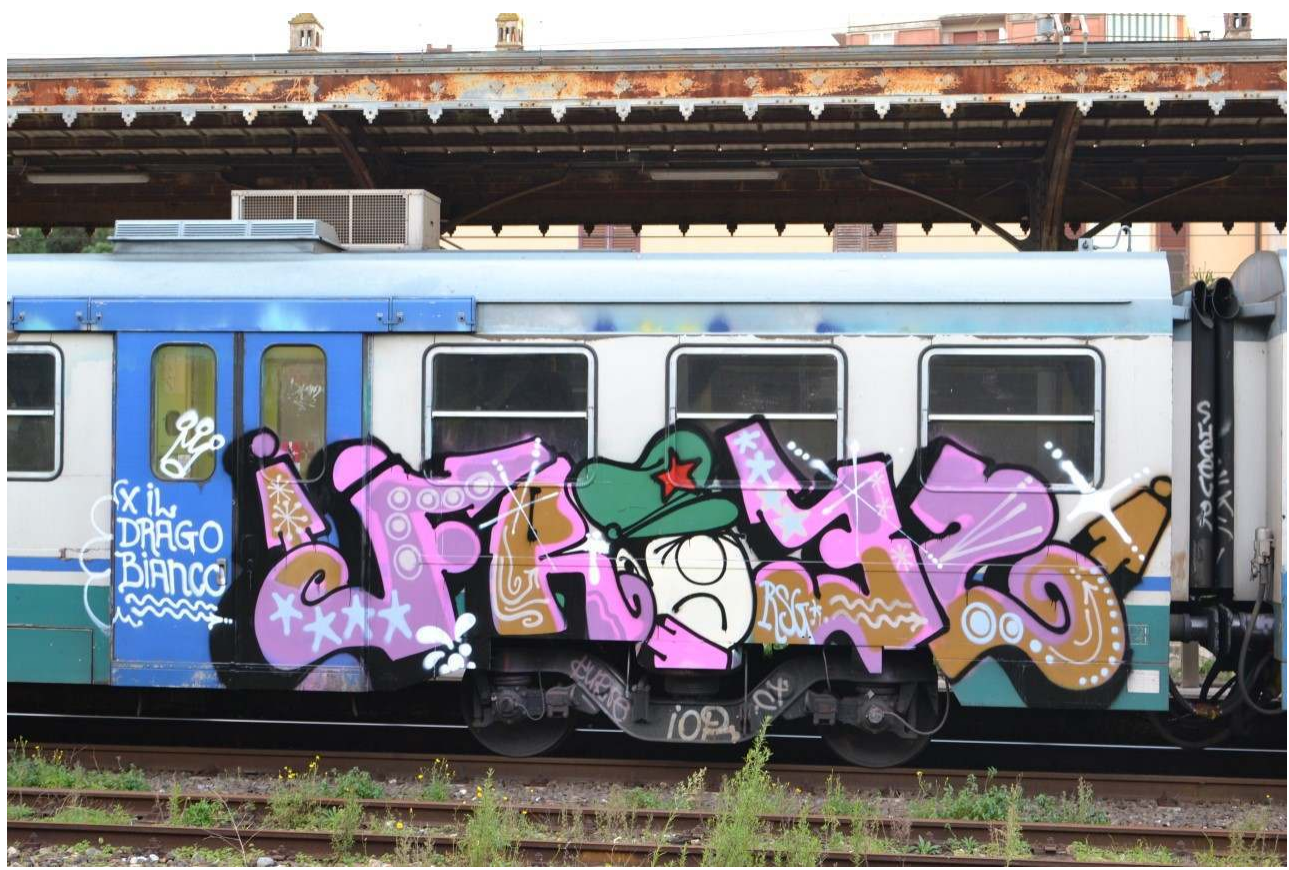

«Graffiti créé par Fra32. »

Il est essentiel de souligner un point important dans cette section. Le paragraphe précédent indique clairement que nous ne faisons pas de distinction entre le graffiti et le street art. Nous sommes d'avis qu'en tant que genre (parmi de nombreux autres genres, tels que le pochoir urbain, les installations d'art urbain, la sculpture urbaine et le tricot urbain, etc.), le graffiti appartient à la catégorie plus générique du street art. Bien entendu, nous sommes conscients que de nombreux théoriciens et praticiens font une distinction entre ces pratiques. Nous ne sommes pas d'accord avec eux. Bien que nous ne réfutions pas leurs différences, les deux pratiques paraissent converger en ce qui concerne leur identification respective avec l'espace public: elles sont toutes deux carnavalesques et, de ce fait, subversives ${ }^{20}$. En d'autres termes, tout comme ceux que nous considérons ici comme des artistes de street art, les graffiti-artists détournent les surfaces visibles du paysage urbain et remodèlent leurs usages et fonctions. Ils transforment principalement ces surfaces recouvertes d'affiches publicitaires en toiles d'expression libre.

Reconnaitre la nature carnavalesque du street art peut permettre de mieux qualifier les possibilités de résistance sociale qui lui sont propres. Comme l'explique de Goede (2005), le carnavalesque joue un rôle significatif dans les stratégies dissidentes contemporaines. $\mathrm{Au}$ lieu de servir de soupapes d'échappement inoffensives, ces stratégies ridiculisent les pratiques d'oppression, afin de défier leur légitimité en exposant leur banalité. En effet, les interventions carnavalesques utilisent le rire ou l'absurdité pour saboter le banal, exposant ainsi son inutilité. Dès que le banal est affiché comme un objet inutile issu de la volonté humaine, il s'ouvre au changement. Par conséquent, en créant un espace dans lequel les catégories usuelles de l'ordre et de la hiérarchie sont mises entre parenthèses, 
le carnaval favorise la création d'une politique alternative imaginaire : celle d'un « autre monde », où les normes et les pratiques sont différentes (Scott 1990).

À travers son intrusion carnavalesque pleine d'ironie et de ridicule, le street art sabote les normes et les conventions qui réglementent les pratiques de visibilité dans les espaces publics. Il se moque du régime d'entreprise et prouve que ces normes et conventions sont loin d'être nécessaires ou inévitables, par le biais de sa présence contestée. Au contraire, le régime d'entreprise, c'est-à-dire la hiérarchie qui donne préséance à la publicité dans les espaces publics, est un produit historique banal de la cooptation capitaliste. Le street art est la preuve visible de la possibilité d'utiliser la rue d'une façon qui ne correspond pas à la logique du profit - car le street art est un « cadeau ${ }^{21}$ »; il prouve également que les individus et les groupes marginalisés, les sous-cultures, et les contre-publics, généralement bannis par la hiérarchie de la visibilité dans les espaces publics, peuvent mettre toutes les chances de leur côtééc ${ }^{22}$ Ainsi, le street art nous permet d'imaginer un autre système de visibilité dans les espaces publics: un système potentiellement plus démocratique et inclusif. Cependant, Foucault affirme que «la transformation devien [dra] à la fois très urgente, très difficile et tout à fait possible ${ }^{23}$ ", si nous pouvons imaginer des solutions alternatives.

Avant de conclure cette section, il nous faut étudier un autre point. Il n'est pas nécessaire que le street art soit illégal pour défier le régime d'entreprise. Que le street art soit souvent illégal, est sans doute plus une caractéristique secondaire de cet art, qu'une caractéristique nécessaire à son identité. En effet, il est important de se souvenir que la visibilité au sein des espaces publics n'est pas uniquement réglementée par les lois positives officielles, mais également par les normes et les conventions sociales officieuses. Nous avons également choisi de citer la notion du partage du sensible de Rancière, car il a conscience de cette nuance. Si l'illégalité occasionnelle des œuvres de street art contribue à leur conférer un pouvoir subversif, il est également possible que des œuvres d'art autorisées défient les hiérarchies dominantes de la visibilité en perturbant les conventions officieuses. Prenons l'exemple du projet Under Art Construction imaginé par Stefan Mohr, un travailleur social basé à Francfort (Baldini 2015). Il inclut les œuvres installées sur la clôture entourant le site de construction du nouveau siège de la Banque Centrale Européenne (BCE), à Francfort. Bien qu'elles soient dûment autorisées et partiellement parrainées par la BCE, ces œuvres défient ouvertement le monopole du pouvoir des entreprises sur la visibilité dans les quartiers financiers. En introduisant des éléments acerbes de critique politique à l'encontre des autorités financières, ces œuvres s'approprient cet espace et redéfinissent ses usages. 


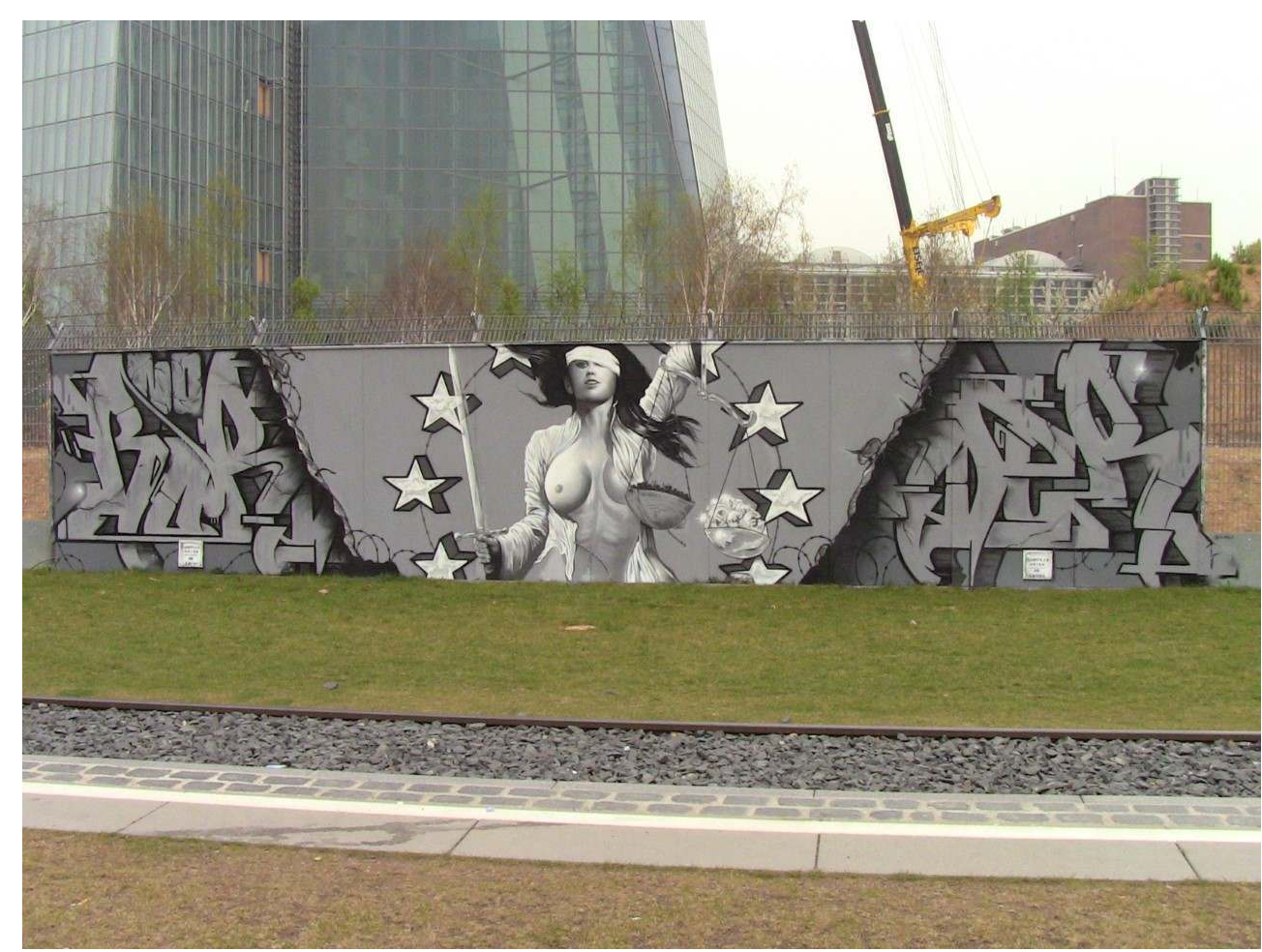

«COR et Bobby Borderline, Lady Justice. »

À l'instar des fous de Shakespeare qui œuvrent pour le roi, les artistes du street art n'ont pas peur de s'exprimer (et ne doivent pas avoir peur), même lorsque leurs activités sont légales.

\section{Conclusion}

À quel moment une œuvre d'art devient-elle une œuvre de street art ? Dans cet article, nous avons suggéré qu'une œuvre d'art devenait une œuvre de street art, si elle défiait, par le biais de stratégies carnavalesques, ce que nous avons appelé le régime d'entreprise - c'est-à-dire, ces lois et conventions qui réglementent la visibilité dans la ville moderne. Nous définissons donc le street art en fonction du rôle qu'il joue dans le conflit politique autour de l'espace public et de ses usages légitimes. Le street art transforme la ville en un espace contesté et se réapproprie également les surfaces urbaines visibles, réservées à un usage commercial. Ce faisant, nous suivons un « courant pragmatique " récent (Baldini 2016) de la philosophie du street art. En participant à la lutte politique pour la visibilité et l'expression libre dans les espaces publics, le street art nous rappelle le mécontentement des pragmatistes envers l'étroitesse des approches modernes de la philosophie de l'art. Au lieu d'occuper un champ distinct de contemplation désintéressée, le street art « fait partie intégrante de la philosophie et des institutions de la communauté ${ }^{4} »$. En contribuant à influencer la nature de l'espace public, il rétablit, dans le sillage de John Dewey, une « continuité entre les ... œuvres d'art et les événements, actes et souffrances $\mathrm{du}$ quotidien ${ }^{25} »$. Le but du street art n'est pas uniquement d'introduire l'art dans les espaces publics. Il s'agit plutôt d'une tentative créative de se réapproprier la ville, une rue à la fois. 


\section{BIBLIOGRAPHY}

Abbey-Lambertz, K. (2012) « Death To Street Art? ». In : The Huffington Post. http://

www.huffingtonpost.com/2012/05/11/detroit-beautification-project-sever-death-to-street-artmural-hamtramck-michigan_n_1508123.html. [3.11.15]

Baker, L. E. (2007) «Public Sites versus Public Sights : The Progressive Response to Outdoor Advertising and the Commercialization of Public Space ». In : American Quarterly, 59.4, 1187-1213.

Bakhtin, M. (1984) Rabelais and His World. Cambridge, MA : MIT Press.

Baldini, A. (2016) « Street Art : A Reply to Riggle ». In : The Journal of Aesthetics and Art Criticism, 74.2, 187-191.

(2015) «An Urban Carnival on the City Walls : The Visual Representation of Financial Power in European Street Art ». In Journal of Visual Culture, 14.2, 246-52.

Banksy. (2005) Wall and Piece. London : Century.

Bartolomeus, L. (2012) «Street Art in Indonesian Social and Political Life. » (2015) Guggenheim Blogs. http://blogs.guggenheim.org/map/street-art-in-indonesian-social-and-political-life/. [30.3.15]

Baudrillard, J. (1981) Simulacra et Simulation. Paris : Galilée.

Bengtsen, P. (2015) « Carelessness or Curatorial Chutzpah ? On Controversies Surrounding Street Art in the Museum ». In : Konsthistorisk tidskrift/Journal of Art History, 84.4, 220-33.

Brighenti, A. (2007) «Visibility A Category for the Social Sciences ». In Current Sociology, 55.3, 32342.

(2010) « At the Wall : Graffiti Writers, Urban Territoriality, and the Public Domain ». In : Space and Culture, 13.3, 315-32.

Campos, R. (2013) « Graffiti Writer as Superhero ». In : European Journal of Cultural Studies, 16.2, $155-70$.

de Certeau, M., et Luce Giard (1998) Culture in the Plural. Minneapolis : University of Minnesota Press.

Confino, J. (2014) «'Fake Street Art Sucks’ : Perrier Replaces Williamsburg's Nelson Mandela Mural ». In : The Guardian. http://www.theguardian.com/sustainable-business/2014/sep/26/ fake-street-art-sucks-perrier-replaces-williamsburgs-nelson-mandela-mural-with-hugeadvertisement. [30.5.15]

Cooke, R. (2005) « Bold, Brave, Beautiful ». In : The Guardian. http://www.theguardian.com/ artanddesign/2005/sep/18/art. [15.05.15]

Dewey, J. (2005) Art as Experience. New York : Berkley Pub. Group.

Dickie, G. (1989) « The New Institutional Theory of Art ». In Aesthetics : A Critical Anthology, New York : St. Martin's Press, 196-205.

Ferrell, J. (1996) Crimes of Style. Urban Graffiti and the Politics of Criminality. Boston, MA: Northeastern University Press. 
Foucault, M. (1981/2001) « Est-il donc important de penser ?». In : Dits et écrits, Tome II : 1976-1986. Paris: Gallimard, 997-1001

de Goede, M. (2005) « Carnival of Money. Politics of Dissent in an Era of Globalizing Finance ». In The Global Resistance Reader. London and New York : Routledge, 379-91.

Halsey, M., et Alison Young. (2006) « 'Our Desires Are Ungovernable' Writing Graffiti in Urban Space ». In : Theoretical Criminology, 10.3, 275-306.

Hamdy, B., et Stone. (2014) Walls of Freedom : Street Art of the Egyptian Revolution. Berlin, Germany : From Here To Fame Publishing.

Irvine, M. (2012) « The Work on the Street : Street Art and Visual Culture ». In : The Handbook of Visual Culture, London : Berg, 235-78.

Iveson, K. (2012) « Branded Cities : Outdoor Advertising, Urban Governance, and the Outdoor Media Landscape ». In : Antipode, 44.1, 151-74.

Margolis, J. (2010) « The Importance of Being Earnest about the Definition and Metaphysics of Art ». In : The Journal of Aesthetics and Art Criticism, 68.3, 215-23.

Matravers, D. (2000) « The Institutional Theory : A Protean Creature ». In : The British Journal of Aesthetics, 40.2, 242-50.

Mattelart, A. (1991) Advertising International : The Privatization of Public Space. London et New York: Routledge.

Pan, L. (2014) « Writing at the End of History : Reflections on Two Cases of Graffiti in Hong Kong ». In : Public Art Dialogue, 4.1, 147-66.

Rancière, J. (1995) La mésentente : Politique et philosophie. Paris : Galilée.

-—-. 2000. Le partage du sensible : esthétique et politique. Paris : La Fabrique-éditions.

Riggle, N. A. (2010) «Street Art: The Transfiguration of the Commonplaces ». In : The Journal of Aesthetics and Art Criticism, 68.3, 243-57.

ryanbyrne123. (Mars 23, 2015) «Political Street Art and Protest ». In : Street Art in Cape Town. https://wallsofcapetown.wordpress.com/2015/03/23/political-street-art-and-protest/. [31.10.15]

Scott, J. C. (1990) Domination and the Arts of Resistance : Hidden Transcripts. New Haven : Yale University Press.

Shusterman, R. (2000) Pragmatist Aesthetics : Living Beauty, Rethinking Art. Lanham, Md: Rowman \& Littlefield.

Simoes, P. (2008) « Vespa Ads Not Cool ». In : Spacing Toronto. http://spacing.ca/

toronto/2008/04/30/vespa-ads-not-cool/. [30.05.15]

Siregar, L. (2012) « Graffiti Artists Document Indonesia's Street Art Online. » In : The Jakarta Globe. http://thejakartaglobe.beritasatu.com/archive/graffiti-artists-document-indonesias-street-artonline/. [30.3.15]

Sommer, D. (2014) The Work of Art in the World: Civic Agency and Public Humanities. Durham : Duke University Press.

Thibault, A. (2014) « Beijing's Thriving Graffiti Culture May Surprise You ». In : Public Radio International. http://www.pri.org/stories/2014-04-21/beijings-thriving-graffiti-culture-maysurprise-you. [25.3.15] 
« Urban Dictionary : Street Art. » (2015). http://www.urbandictionary.com/define.php? term=street+art. [13.06.15]

Valjakka, M. (2013) « Graffiti in China - Chinese Graffiti ?» The Copenhagen Journal of Asian Studies, 29.1, 61-91.

Vartanian, H. (2010) « Street Art Politics \& Commercialization... How Far Is Too Far ? » In : Hyperallergic. http://hyperallergic.com/3415/street-art-politics-commercialization/. [30.5.15]

Volodzko, D. (2015) « Graffiti in China, Part Two : The Writing on the Wall ». In : The Diplomat. http://thediplomat.com/2015/05/graffiti-in-china-part-two-the-writing-on-the-wall/. [12.6,15]

Walker, R. (2008) « Vespa Murketing Inspires Fauxreel Backlash?». In : Murketing [The Journal Of]. http://www.murketing.com/journal/?p=1310. [30.5.15]

Young, A. (2014) Street Art, Public City : Law, Crime and the Urban Imagination. Abingdon, Oxon: Routledge.

Zangwill, N. (2007) Aesthetic Creation. Oxford : Oxford University Press.

\section{APPENDIXES}

\section{Liste des images}

FIG. 1 : omino 71. (Novembre 9, 2014) etnik. https://www.flickr.com/photos/ omino $71 / 15963513240 /$

FIG. 2 : Fra32. (Novembre 26, 2015) Fra32.

FIG. 3 : Baldini, A. (Mars 31, 2014) Lady Justice by COR and Bobby Borderline.

\section{NOTES}

1. La théorie institutionnelle de l'art (Dickie 1989) est un exemple renommé de définition classificatoire de l'art.

2. L'un des nombreux défenseurs d'une définition évaluative intéressante du street art est Zangwill (2007).

3. Rancière, J. (2000) Le partage du sensible: esthétique et politique. Paris : La Fabrique-éditions.

4. Sommer (2014) parle d'une convergence entre les esthétiques pragmatiques (de Dewey, notamment) et l'esthétique de Rancière.

5. Cf. Baudrillard, J. (1981) Simulacra et Simulation. Paris : Galilée, p. 133.

6. Irvine, M. (2012) «The Work on the Street: Street Art and Visual Culture ». In : The Handbook of Visual Culture, London : Berg, p. 251.

7. Baldini (2016) traite également ces questions.

8. Irvine, M. (2012) « The Work on the Street: Street Art and Visual Culture ». In : The Handbook of Visual Culture, London: Berg, p. 251.

9. Banksy. (2005) Wall and Piece. London: Century, p. 9.

10. Confino, J. (2014) «'Fake Street Art Sucks': Perrier Replaces Williamsburg's Nelson Mandela Mural ». In : The Guardian.

11. Ibid.

12. Volodzko, D. (2015) « Graffiti in China, Part Two: The Writing on the Wall ». In : The Diplomat.

13. Baldini (2016) offre une critique de Riggle (2010). 
14. Portons rapidement notre attention sur une question qu'il nous sera impossible de développer entièrement dans cet article. Contrairement à Riggle, nous estimons qu'il est possible de trouver des œuvres de street art dans des espaces officiellement consacrés à l'art, pourvu qu'elles défient le partage du sensible qui réglemente la visibilité dans ces contextes, par le biais de stratégies carnavalesques. Par exemple, il est possible que les œuvres introduites illégalement par Banksy dans des musées soient des œuvres de street art. Pareillement, il est parfaitement possible de trouver des œuvres de street art dans des lieux autres que la rue, les places publiques et les gares. L'installation illégale d'une œuvre de Banksy à Disneyland en est un exemple parfait. 15. Cooke, R. (2005) “Bold, Brave, Beautiful”. In: The Guardian.

16. Bakhtin, M. (1984) Rabelais and His World. Cambridge, MA: MIT Press, p. 91.

17. Curieusement, les graffiti-artists appellent leurs collègues les plus talentueux des « rois ».

18. Campos, R. (2013) “Graffiti Writer as Superhero". In: European Journal of Cultural Studies, 16.2, p. 155.

19. Brighenti, A. (2010) «At the Wall: Graffiti Writers, Urban Territoriality, and the Public Domain ». In: Space and Culture, 13.3, p. 321.

20. Certains suggèrent que l'utilisation d'un terme plus neutre, tel « art urbain », pourrait mieux nous permettre de désigner ces pratiques. Nous nous opposons à cette proposition pour les raisons suivantes. Premièrement, il est probable que l'art urbain inclut également les œuvres d'art public officiel, installées dans les environnements urbains. Comme expliqué ci-dessus, le problème, dans ce cas, réside dans le fait que le street art et le graffiti fonctionnent différemment de leurs contreparties officielles. Deuxièmement, de nombreux spectateurs désignent tous les styles et toutes les pratiques (y compris le graffiti, le pochoir urbain, les installations artistiques urbaines, etc.) que nous étudions dans le présent article sous le nom de "street art ", dans la langue usuelle. Compte tenu de l'objectif de cet article, qui est d'informer le débat actuel concernant les problèmes en question, nous préférons éviter l'expression ad hoc, qui pourrait très bien aliéner les non-spécialistes du débat.

21. Irvine, M. (2012) “The Work on the Street: Street Art and Visual Culture”. In: The Handbook of Visual Culture, London: Berg, p. 250.

22. Rancière (1995, p. 23) et Brighenti (2007, p. 329) argumentent que la marginalisation sociale et politique est une fonction de l'exclusion de certaines personnes de l'espace visible dans les espaces publics.

23. Foucault, M. (1981/2001) «Est-il donc important de penser ? Domain ». In : Dits et écrits, Tome II : 1976--1986. Paris : Gallimard, 1000.

24. Dewey, J. (2005) Art as Experience. New York: Berkley Pub. Group, p. 8

25. Ibid., p. 3.

\section{ABSTRACTS}

This paper develops a pragmatist definition of street art acknowledging its essential subversiveness, while drawing implications at the level of street art's political and social function. By developing an insight from Irvine (2012), the paper argues that street artworks turn inside out accepted norms of visibility in public spaces. Street artists challenge those norms by deploying carnivalesque strategies such as irony and misappropriation (Scott 1990). In this sense, street art is art intrinsically challenging dominant hierarchies of visibility through the use of 
carnivalesque strategies. By opposing advertising's dominion over the city walls, they reclaim - one street at a time - the possibility of expressing themselves in public for those generally excluded.

L'article propose une définition pragmatiste du street art, qui tient compte de sa subversivité essentielle et expose les implications des rôles politique et social de cette forme artistique. En développant la théorie d'Irvine (2012), l'article soutient que les œuvres de street art ont entièrement détourné les normes de visibilité dans les espaces publics. Les artistes de street art défient ces normes en se servant de stratégies carnavalesques, tels l'ironie et le détournement (Scott 1990). Dans ce sens, le caractère intrinsèque du street art réside dans la volonté de défier la hiérarchie dominante de la visibilité, à l'aide de stratégies carnavalesques. En s'opposant à la présence dominatrice de la publicité sur les murs des espaces urbains, les œuvres de street art revendiquent, une rue à la fois, la possibilité de s'exprimer en public pour ceux qui n'en ont généralement pas le droit.

\section{INDEX}

Chronological index: XXIe siècle

Geographical index: République populaire de Chine

Keywords: advertising, art, politics, Bakhtin, carnival, Rancière, social resistance, street art

Mots-clés: art, politique, Bakhtin, carnaval, Rancière, publicité, résistance sociale, street art

\section{AUTHOR}

\section{ANDREA BALDINI}

Andrea Baldini est chercheur postdoctoral au sein des institutions chinoises Institute for Advanced Studies in Humanities and Social Sciences (IAS) et Art Institute de Nanjing University. Il détient un doctorat en philosophie de l'université Temple University (Philadelphie, États-Unis) et un doctorat en sciences textuelles de l'Université de Sienne (Italie). Ses intérêts de recherche portent sur la philosophie de l'art, la culture visuelle, l'espace public et les valeurs non esthétiques de l'art. Il est l'auteur de nombreuses publications sur les thèmes de l'art public, du street art et de la philosophie de la musique. 